\title{
Magnetic and Electronic Properties of Selected Rare-Earth Chromium Germanides Compounds
}

\author{
A. GIL ${ }^{a, *}$ AND M. MAKOWSKA-JANUSIK ${ }^{b}$ \\ ${ }^{a}$ Department of Mathematics and Natural Sciences, Jan Długosz University in Częstochowa, \\ al. Armii Krajowej 13/15, 42-200 Częstochowa, Poland
}

${ }^{b}$ Institute of Physics, Jan Długosz University in Częstochowa, al. Armii Krajowej 13/15, 42-200 Częstochowa, Poland

The ternary rare-earth compounds exhibit interesting physical and structural properties. These structures changing composition and stoichiometry change drastically their magnetic properties. Some of them, based on chromium germanides were studied experimentally concerning their magnetic properties and up to our knowledge they were never studied theoretically explaining nature of their magnetic behaviour. In the presented work the nature and mechanism of the magnetic and electronic properties of rare-earth chromium germanides is presented. The magnetic and electronic properties of $\mathrm{RCrGe}_{2}$ and $\mathrm{RCr}_{0.3} \mathrm{Ge}_{2}(\mathrm{R}=\mathrm{Tb}, \mathrm{Dy}, \mathrm{Ho}$ or Er) were investigated theoretically applying plane-wave density functional theory/Perdew-Burke-Ernzerhof methodology. The computational investigations were performed for the stoichiometric and nonstoichiometric crystal structure with the space group $\mathrm{Cmcm}$. The stoichiometry of the rare-earth chromium germanides compounds has significant effect on the magnetic properties of the investigated material. The theoretical predictions are compared to the experimentally obtained results.

DOI: $10.12693 /$ APhysPolA.127.385

PACS: 75.30.Fv, 75.47.Np, 71.15.Mb, 71.15.Ap

\section{Introduction}

The rare-earth chromium germanides $\mathrm{RCr}_{0.3} \mathrm{Ge}_{2}$ $(\mathrm{R}=\mathrm{Tb}, \mathrm{Dy}, \mathrm{Ho}$ or Er) were studied experimentally concerning their magnetic properties [1]. There presented results confirm the orthorhombic $\mathrm{CeNiSi}_{2}$-type crystal structure (space group $\mathrm{Cmcm}$ ) and the antiferromagnetic ordering at low temperatures. The change of their magnetic type structure from the collinear commensurate for $\mathrm{R}=\mathrm{Tb}$, Dy and Ho to the modulated incommensurate for $\mathrm{R}=\mathrm{Er}$ is observed. In general, the magnetic ordering in the studied compounds originates from interplay of exchange interactions between the magnetic moments localized on the rare-earth atoms, which are arranged in double-layer slabs stacked along the $b$-axis. The $\mathrm{R}-\mathrm{R}$ distances within the slabs are near to $4.0 \AA$, whereas those between the slabs are $\approx 5.5 \AA$. The rareearth moments couple indirectly by spin polarization of conduction electrons (the Ruderman-Kittel-KasuyaYosida (RKKY) mechanism). The quantum chemical calculations may be helpful to explain structural depending changes appearing at the magnetic properties of mentioned materials. The chromium germanides structures by changing composition and stoichiometry change drastically their magnetic properties [2, 3].

In presented work the magnetic and electronic properties of rare-earth chromium germanides like $\mathrm{RCrGe}_{2}$ and $\mathrm{RCr}_{0.3} \mathrm{Ge}_{2}(\mathrm{R}=\mathrm{Tb}, \mathrm{Dy}, \mathrm{Ho}$ or Er) will be investigated theoretically applying plane-wave density functional theory/Perdew-Burke-Ernzerhof (DFT/PBE) methodology. The theoretical predictions will be compared to the experimentally obtained results. The sto-

*corresponding author; e-mail: a.gil@ajd.czest.pl

ichiometry of the rare-earth chromium germanides compounds has significant effect on the magnetic properties of compounds.

\section{Details of quantum chemical calculations}

To compute the magnetic properties of selected chromium germanides first of all their crystal structure was built. As the starting single crystal the stoichiometric $\mathrm{RCrGe}_{2}$ composition was chosen. All the investigated structures are orthorhombic with space group Cmcm [1,4]. Their crystal parameters as well as the atomic positions were defined as it is presented in Ref. [1] and the crystal structures of studied materials were built using Materials Studio package [5]. Such formed unit cells of the $\mathrm{DyCrGe}_{2}$ is presented in Fig. 1. The other investigated structures based on $\mathrm{Tb}, \mathrm{Ho}$ and $\mathrm{Er}$ have very similar look. Instead dysprosium they possess $\mathrm{Tb}, \mathrm{Ho}$ or Er atoms, respectively. Also the volume of investigated structures are similar as presented in Ref. [1].

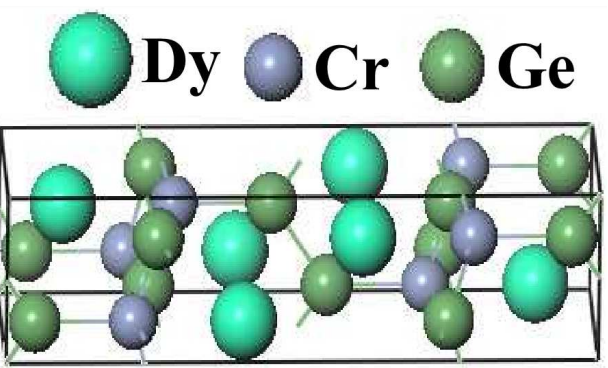

Fig. 1. The unit cell of $\mathrm{DyCrGe}_{2}$ crystal structure.

Before to start quantum chemical calculations of magnetic properties of the bulk crystals their geometry was optimised. The procedure was performed applying DFT via the CASTEP program [6] based on total energy plane-wave pseudopotential methodology. The geometries of all investigated structures were optimised 
with respect to the total energy minimization applying the Broyden-Fletcher-Goldfarb-Shanno (BFGS) algorithm [7]. The unit cell size and the symmetry of crystals were conserved during the geometry optimisation procedure. The convergence criteria for optimization were chosen as follows: the convergence of total energy during geometry optimization procedure equal to $10^{-5} \mathrm{eV}$ /atom, force on the atom was less than $0.03 \mathrm{eV} / \AA$, the stress on the atom was not more than $0.05 \mathrm{GPa}$, the maximal atomic displacement was equal to $10^{-3} \AA$. The electronic exchange-correlation energy was treated within the frame of the GGA approximation using PBE [8] potentials. The relativistic effect was included in the performer calculations at the level of the scalar-relativistic zeroth-order regular approximation (ZORA) [9]. In order to allow performance of calculations with the lowest possible cut-off energy for the plane-wave basis set, norm-conserving pseudopotentials have been used in the calculations [10]. Pseudoatomic calculations were performed for $\mathrm{Cr} 3 d^{5} 4 s^{1}$, Ge $4 s^{2} 4 p^{2}$, Tb $4 f^{9} 5 s^{2} 5 p^{6} 6 s^{2}$, Dy $4 f^{10} 5 s^{2} 5 p^{6} 6 s^{2}$, Ho $4 f^{11} 5 s^{2} 5 p^{6} 6 s^{2}$, and Er $4 f^{12} 5 s^{2} 5 p^{6} 6 s^{2}$ configurations. The cut-off energy of the plane-wave basis set was chosen equal to $310 \mathrm{eV}$. The special points numerical sampling integration over the Brillouin zone are carried out using the MonkhorstPack method with a $7 \times 2 \times 7$ special k-point mesh. The total energy is assumed to be converged when the SCF tolerance is $10^{-5} \mathrm{eV}$ /atom. The calculations were performed for a primitive unit cell of each structure with spin-unrestricted regime. The initial spin for the unpaired electrons for each atom was taken from the formal spin specified for each atom then the starting value was subsequently optimized during the calculation.

The nonstoichiometric $\mathrm{RCr}_{0.3} \mathrm{Ge}_{2}$ structures were constructed starting from stoichiometric unit cell. Consequently subsequent chromium atoms were removed keeping the nonstoichiometry of structure as $\mathrm{RCr}_{0.3} \mathrm{Ge}_{2}$. In each step of removing of chromium atom the geometry of new obtained structure was optimised. For all obtained structures their total energy was calculated and they were compared one to the other. The nonstoichiometric structure with the lowest total energy was chosen as the one which was taken for the essential calculations. The quantum chemical parameters were chosen as described above. The magnetic properties were computed for both stoichiometric as well as nonstoichiometric crystal structures.

\section{Results and discussion}

The rare-earth chromium germanides do not crystallise in stoichiometric structure but to start computer simulations the well defined geometry is necessary. This is way, first of all the stoichiometric crystals were investigated and then $\mathrm{Cr}$ vacancies were created. The position of vacancies was chosen according to the total energy minimum.

Following the geometry optimization, the theoretically obtained structural parameters are in good agreement with the data contained in work of Bie et al. [4]. The parameters of all investigated structures are very similar. Comparing the results from Table I, Ref. [1] to the simulated data, one may see that the biggest volume has the unit cell of $\mathrm{TbCrGe}_{2}$ structure and the smallest one possesses $\mathrm{HoCrGe}_{2}$ and $\mathrm{ErCrGe}_{2}$. It is according to the "crystal" radius of rare-earth atoms. The "crystal" radius of $\mathrm{Tb}$ is equal to $1.18 \AA$, for the Ho and Er they are $1.04 \AA$ and $1.03 \AA$, respectively [11].

Performed simulations confirm that the rare-earth atoms are arranged in double-layer slabs and they have essential impact on the magnetic properties of investigated compounds.

In Fig. 2 the spin density isosurfaces calculated by $\mathrm{DFT} / \mathrm{PBE}$ are presented. One may see that the most high spin density is located at the rare-earth atoms. In the case of $\mathrm{DyCrGe} 2, \mathrm{HoCrGe}_{2}$ and $\mathrm{ErCrGe}_{2}$ structures the two surfaces of rare-earth atoms located at double-layered atomic part have antiparallel spin. One layer of atoms has spin against the surface of figure and the other one at the surface. The situation is completely different for the $\mathrm{TbCrGe}_{2}$ structure. At very low temperature of simulations this material has ferromagnetic nature because two layers of $\mathrm{Tb}$ atoms possess parallel spin. The same small contribution to the magnetism of all investigated structure comes from the $\mathrm{Cr}$ atoms.
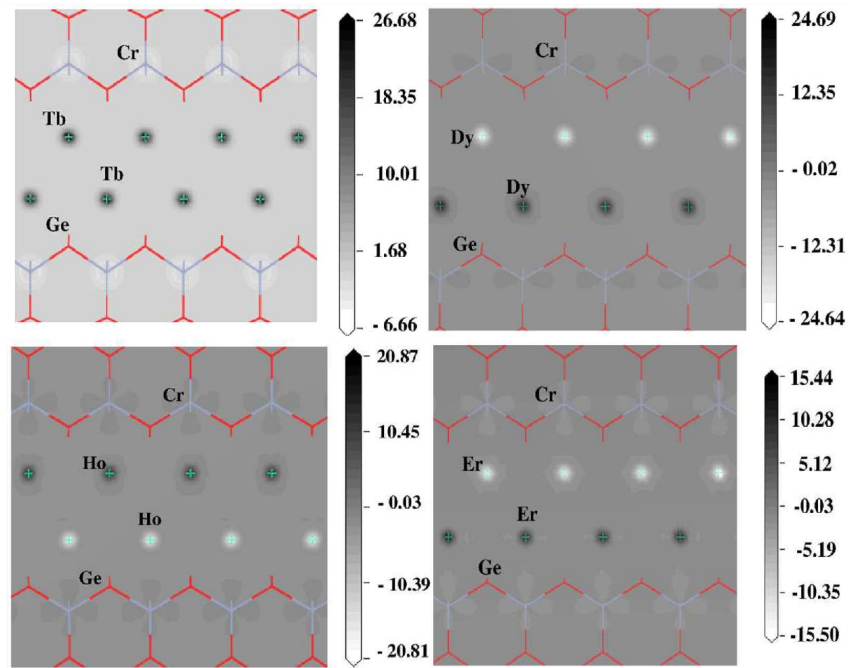

Fig. 2. The spin-density distribution calculated by DFT/PBE methodology for the $\mathrm{TbCrGe}{ }_{2}, \mathrm{DyCrGe}_{2}$, $\mathrm{HoCrGe}_{2}$, and $\mathrm{ErCrGe}_{2}$.

In Fig. 3 one may see the most important electron population located at the rare-earth atoms. It is also observed that for the stoichiometric structure the important charge is located at the $\mathrm{Cr}$ atoms (see Fig. 3, $\mathrm{DyCrGe} 2$ - orange part). In the case of stoichiometric structure the rare-earth atoms and $\mathrm{Cr}$ possess spin-unpaired electrons which is seen in Fig. 2. It gives conclusion that in $\mathrm{RCrGe}_{2}$ stoichiometric crystals the Ge-electrons are shifted to the $\mathrm{Cr}$ atoms. Concerning electron localization the $\mathrm{DyCrGe}_{2}$ and $\mathrm{HoCrGe}_{2}$ material are very similar. For these two structures the localization of electron wave functions is seen between two layers of Dy or Ho atoms. For the $\mathrm{TbCrGe}_{2}$ and $\mathrm{ErCrGe}_{2}$ structure the electrons belonging to the rare-earth atoms are localised at these atoms without so fare splitting as it is observed in the case of Dy and Ho. In case of the $\mathrm{ErCrGe}_{2}$ structure the electrons localization is more pronounced for the $\mathrm{Ge}$ than for $\mathrm{Cr}$ atoms but the electron located at Ge atom are paired. The electrons of $\mathrm{Tb}$ atoms in stoichiometric crystal are not affected by the neighbour layer of $\mathrm{Cr}$ and $\mathrm{Ge}$ atoms. The $\mathrm{Tb}$ electrons are closely related to the core of atom while the spins are parallel for two neighbouring layers. 


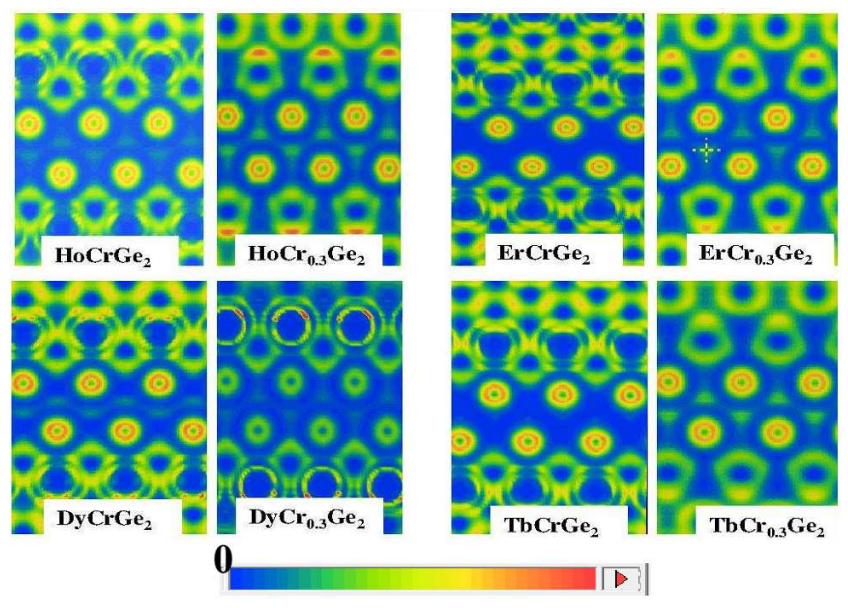

Fig. 3. Electron localization calculated by DFT/PBE methodology for stoichiometric $\mathrm{RCrGe}_{2}$ and nonstoichiometric $\mathrm{RCr}_{0.3} \mathrm{Ge}_{2}$ structures.

In the case of the nonstoichiometric structures the electrons are localized at rare-earth atoms as well as at the Ge but the ones located at rare-earth atoms are unpaired and the Ge electrons are paired. All nonstoichiometric structures possess the same electrons diffused between the layers of rare-earth atoms. It is very significant for the $\mathrm{TbCr}_{0.3} \mathrm{Ge}_{2}$ structure. Probably, through interatomic interaction the electrons from $\mathrm{Cr}$ and $\mathrm{Ge}$ atoms are delocalised and shifted more in the area of $\mathrm{Tb}$. It may be reason of the antiparallel orientation of spin located at the $\mathrm{Tb}$. The magnetic behaviour of the nonstoichiometric $\mathrm{RCr}_{0.3} \mathrm{Ge}_{2}$ structures is caused by the geometrical changes according to the total energy minimization of the crystal. The creation of nonstoichiometric $\mathrm{RCr}_{0.3} \mathrm{Ge}_{2}$ crystals change the distances between atoms comparing to the stoichiometric material. The rareearth atoms located in double-layer slices are closer one to the other than it was for stoichiometric structure and simultaneously the $\mathrm{R}$ atoms are more far from the $\mathrm{Cr}$ atoms. Thus, for the nonstoichiometric compounds their magnetism originates from the magnetic moments of $f$ electrons localized on the $\mathrm{R}$ atoms and the $d$ electrons of $\mathrm{Cr}$ do not contribute to the magnetic properties of the crystals as it was for the stoichiometric structure.

In Fig. 4 the calculated spin density of the nonstoichiometric structure is presented. One may see that for the $\mathrm{DyCr}_{0.3} \mathrm{Ge}_{2}$ structure the spin density localized at Dy atoms did not change. Again the rare-earth atoms play the main role in magnetism of the investigated material. Any spin density is not seen at the $\mathrm{Cr}$ atoms. The magnetic properties of $\mathrm{Cr}$ uncompleted systems are different than for stoichiometric crystal structures. Analysing Fig. 4 one may see that the spin density at the $\mathrm{Tb}$ atoms was changed. The magnetism of non-stoichiometric $\mathrm{TbCr}_{0.3} \mathrm{Ge}_{2}$ structure has the same nature like to behaviour of other crystals. The spin located at the neighbouring layers of $\mathrm{Tb}$ has opposite direction.

\section{Conclusions}

In the presented work the nature and mechanism of the magnetic and electronic properties of $\mathrm{RCrGe}_{2}$ and $\mathrm{RCr}_{0.3} \mathrm{Ge}_{2}$ were investigated theoretically applying planewave DFT/PBE method. The results lead to the following conclusions: the diverse magnetic behaviour originates from the competition of interactions between the $\mathrm{R}$ atoms,
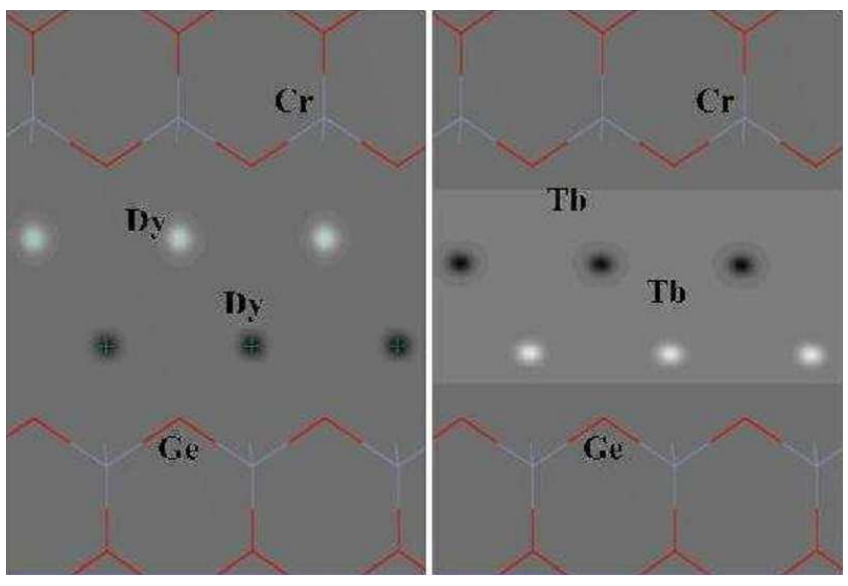

Fig. 4. The spin-density distribution calculated by DFT/PBE methodology for the $\mathrm{DyCr}_{0.3} \mathrm{Ge}_{2}$ (left) and $\mathrm{TbCr}_{0.3} \mathrm{Ge}_{2}$ (right) structures.

which are arranged in double-layer slabs stacked perpendicular to the $b$ axis. In the $\mathrm{Cr}$ reached structures the $d$ electrons on the $\mathrm{Cr}$ atoms provide little to the magnetic properties of crystals. The stoichiometry of the rare-earth chromium germanides compounds has significant effect on the electronic properties of compounds. These findings correlate well with the experimentally obtained results.

\section{Acknowledgments}

Calculations have been carried out in Wrocław Center for Networking and Supercomputing http://www.wcss.wroc.pl (Grant no. 171). The Materials Studio package was used under Poland Country-Wide License.

\section{References}

[1] A. Gil, D. Kaczorowski, B. Penc, A. Hoser, A. Szytula, J. Solid State Chem. 184, 227 (2011).

[2] A.V. Morozkin, R. Nirmala, J. Yao, Y. Mozharivskyj, O. Isnard, J. Solid State Chem. 196, 93 (2012).

[3] M. Yarema, O. Zaremba, R. Gladyshevskii, V. Hlukhyy, T.F. Fassler, J. Solid State Chem 196, 72 (2012).

[4] H. Bie, A.V. Tkachuk, A. Mar, J. Solid State Chem. 182, 122 (2009).

[5] accelrys.com/products/materials-studio/index.html.

[6] S.J. Clark, M.D. Segall, C.J. Pickard, P.J. Hasnip, M.J. Probert, K. Refson, M.C. Payne, Z. Kristallogr. 220, 567 (2005).

[7] B.G. Pfrommer, M. Cote, S.G. Louie, M.L. Cohen, J. Comput. Phys. 131, 233 (1997).

[8] J.P. Perdew, K. Burke, M. Ernzerhof, Phys. Rev. Lett. 77, 3865 (1996).

[9] J.F. Hinton, R.W. Briggs, NMR Period. Table, Eds.: R.K. Harris, B.E. Mann, Academic, London 1978, p. 279; S. Aldridge, Angew. Chem. Int. Ed. 45, 8097 (2006)

[10] D.R. Hamann, M. Schluter, C. Chiang, Phys. Rev. Lett. 43, 1494 (1979).

[11] E. Clementi, D.L. Raimondi, W.P. Reinhardt, J. Chem. Phys. 38, 2686 (1963). 